\title{
Plasma level of lipocalin 2 is increased in neovascular age-related macular degeneration patients, particularly those with macular fibrosis
}

\author{
Mei Chen ${ }^{1}$, Nan Yang', Judith Lechner', Levente Toth', Ruth Hogg ${ }^{2}$, Giuliana Silvestri', Usha Chakravarthy² and \\ Heping $\mathrm{Xu}^{1 *}$ (D)
}

\begin{abstract}
Background: Previously, we and others have reported higher populations of circulating neutrophils in patients with neovascular age-related macular degeneration (nAMD). Neutrophil gelatinase-associated lipocalin (NGAL, also known as lipocalin-2, LCN2), an important innate immune mediator, is known to be critically involved in sterile inflammation-mediated organ failure, fibrosis, cancer progression and retinal degeneration. This study investigated the plasma levels of LCN2, matrix metalloproteinase 9 (MMP9) and LCN2/MMP9 complex in different types of nAMD and examined whether the levels were related to patients' responsiveness to anti-VEGF therapy.

Results: One hundred and seventy-four nAMD patients, including 108 with choroidal neovascularisation (CNV), 32 with retinal angiomatous proliferation (RAP), 23 with polypoidal choroidal vasculopathy (PCV) and 11 unclassified patients, and 43 healthy controls were recruited to this case-control study. Fifty-eight nAMD patients had macular fibrosis and 110 patients did not. Out of the 174 nAMD patients, 80 patients responded completely, 90 responded partially, and 4 did not respond to the anti-VEGF therapy. The plasma levels of LCN2 in nAMD patients (181.46 \pm $73.62 \mathrm{ng} / \mathrm{ml}$ ) was significantly higher than that in healthy controls $(152.24 \pm 49.55 \mathrm{ng} / \mathrm{ml}, P=0.047)$. However, the difference disappeared after adjusting for age. A positive correlation between plasma level of LCN2 and age was observed in nAMD patients $(r=0.29, P=0.0002)$ but not in healthy controls. The plasma level of LCN2 was also positively correlated with circulating neutrophils in nAMD patients $(r=0.34, p=0.0007)$ but not in healthy controls $(r=0.057, p=0.77)$. No correlation was observed between age and circulating neutrophils. Further analysis of nAMD subtypes uncovered a significantly higher level of LCN2 in patients with macular fibrosis even after adjusting for age. No relationship was observed between plasma levels of LCN2 and patients' responsiveness to anti-VEGF therapy. The plasma levels of MMP9 and LCN2/MMP9 complex were comparable between nAMD and controls.

(Continued on next page)
\end{abstract}

\footnotetext{
* Correspondence: heping.xu@qub.ac.uk

${ }^{1}$ Centre for Experimental Medicine, The Wellcome-Wolfson Institute for Experimental Medicine, School of Medicine, Dentistry \& Biomedical Science, Queen's University Belfast, 97 Lisburn Road, Belfast BT9 7BL, UK

Full list of author information is available at the end of the article
}

(c) The Author(s). 2020 Open Access This article is licensed under a Creative Commons Attribution 4.0 International License, which permits use, sharing, adaptation, distribution and reproduction in any medium or format, as long as you give appropriate credit to the original author(s) and the source, provide a link to the Creative Commons licence, and indicate if changes were made. The images or other third party material in this article are included in the article's Creative Commons licence, unless indicated otherwise in a credit line to the material. If material is not included in the article's Creative Commons licence and your intended use is not permitted by statutory regulation or exceeds the permitted use, you will need to obtain permission directly from the copyright holder. To view a copy of this licence, visit http://creativecommons.org/licenses/by/4.0/ The Creative Commons Public Domain Dedication waiver (http://creativecommons.org/publicdomain/zero/1.0/) applies to the data made available in this article, unless otherwise stated in a credit line to the data. 
(Continued from previous page)

Conclusions: Our results suggest that higher plasma levels of LCN2 in nAMD are related to ageing and increased population of circulating neutrophils. Our results also suggest that higher levels of LCN2 may increase the risk of macular fibrosis in nAMD.

Keywords: Age-related macular degeneration, Inflammation, Neutrophils, Lipocalin 2, Matrix metalloproteinase, Macular fibrosis, Plasma

\section{Background}

Age-related macular degeneration (AMD) is the progressive degeneration and loss of function of the central part of the back of the eye, the macula, resulting from old age. AMD is the leading cause of blindness in the elderly in developed countries [1]. There are two advanced forms of AMD, geographic atrophy (GA) and neovascular AMD (nAMD) [2]. nAMD can be further classified into three subtypes, (a) choroidal neovascularisation (CNV), characterised by infiltration of abnormal blood vessels from the choroid into the sub-retinal pigment epithelial (RPE) or sub-retinal space [2]; (b) retinal angiomatous proliferation (RAP) [3], the de novo growth of new blood vessels from retinal vessels that can fuse with $\mathrm{CNV}$; (c) polypoidal choroidal vasculopathy (PCV), sub-RPE infiltration of choroid-derived polypoidal lesions [4]. nAMD account for $80 \%$ of AMD-mediated visual loss [5] and is currently treated with intravitreal injection of vascular endothelial growth factor (VEGF) inhibitors. Although the therapy can stabilise or even improve visual function [6], around a third of patients do not respond to the therapy [7]. Furthermore, approximately $50 \%$ of treated eyes may develop macular fibrosis and those patients may ultimately lose vision [8]. Currently, there is no treatment for GA. Pathogenesis of AMD is not fully understood although inflammation is believed to play an important role $[9,10]$.

Exactly how inflammation leads to macular damage in AMD remains poorly defined. Increased systemic and local inflammatory responses, including complement activation [11-15], immune cell alteration [16-19] and cytokine production [20-24] have been observed in AMD. Previously, we and others have reported increased circulating neutrophils in nAMD patients [19, 25, 26]. Neutrophils are the major type of innate immune cells making up 40-70\% circulating leukocytes. Apart from their anti-microbial functions, neutrophils are also known to participate in various non-infectious sterile inflammations, for example by releasing inflammatory mediators and neutrophil extracellular traps (NETs). The neutrophil gelatinase-associated lipocalin (NGAL, also known as lipocalin-2, LCN2) is constitutively released to the bloodstream by neutrophils under normal physiological conditions [27]. LCN2 binds to the siderophores of bacteria limiting their growth [27]. LCN2 also plays a critical role in iron homeostasis [28], in stabilising matrix metalloproteinase 9 (MMP9) [29], and higher levels of MMP9 is known to be involved in AMD [30]. The production of LCN2 is often increased in disease conditions, and it is known to be involved in sterile inflammation, wound-healing and tissue remodelling, fibrosis [31-34], kidney failure [35, 36], neurodegeneration [37, 38] and cancer progression [39]. Importantly, recent studies have shown that LCN2 actively participates in retinal inflammation and contributes to the development of early and dry AMD [40].

We hypothesized that higher circulating levels of neutrophils may contribute to nAMD through the release of LCN2. LCN2 may participate in nAMD development by promoting dysregulated macular inflammation or stabilizing MMP9. This study aimed to understand if the plasma levels of LCN2, MMP9 and LCN2/MMP9 complex are increased in $\mathrm{nAMD}$ patients and their relationship with circulating neutrophils. Here, we showed that the plasma level of LCN2, but not MMP9 or LCN2/MMP9 complex, was significantly increased in nAMD patients, particular those with macular fibrosis. We further found that the plasma levels of LCN2 positively correlated to age and circulating neutrophils in nAMD but not controls. Our results suggest that LCN2 may play a role in macular fibrosis secondary to nAMD.

\section{Results \\ Demographic and clinical characteristics of participants} A total of 174 nAMD patients and 43 healthy controls were recruited to this study. Demographic analysis showed no difference between the two groups in gender distribution, history of hypertension and cardiovascular disease, history of diabetes and family history of AMD (Table 1). There was also no difference in smoking status, body mass index (BMI), and psychiatry drug intake between nAMD patients and healthy controls. However, the average age when blood was taken, the number of participants who took vitamin supplements or low-dose aspirin were significantly higher in nAMD group compared with those in controls (Table 1). Since vitamin supplementation is usually advised to AMD patients, the higher number of people taking vitamin supplements in the nAMD group is likely the consequence of the disease, rather than a potential contributor to the disease. 
Table 1 Demographic and clinical characteristics of nAMD patients and controls

\begin{tabular}{|c|c|c|c|c|}
\hline & All $(N=217)$ & Control $(N=43)$ & nAMD $(N=174)$ & $P$ value \\
\hline Age, mean (range) & $77.3(53-93)$ & $73.4(58-92)$ & $78.3(53-93)$ & $0.001^{\mathrm{a}}$ \\
\hline Female, number (\%) & $110(51)$ & $19(44)$ & $91(52)$ & $0.341^{b}$ \\
\hline Family history of AMD, number (\%) & $46(21)$ & $6(14)$ & $40(23)$ & $0.210^{\mathrm{b}}$ \\
\hline History of hypertension, number (\%) & $133(62)$ & $23(53)$ & $110(63)$ & $0.312^{b}$ \\
\hline History of diabetes, number (\%) & $28(13)$ & $2(5)$ & $26(15)$ & $0.078^{b}$ \\
\hline History of cardiovascular disease, number (\%) & $57(26)$ & $9(21)$ & $48(28)$ & $0.416^{\mathrm{b}}$ \\
\hline Smoking status & & & & $0.558^{\mathrm{b}}$ \\
\hline Non-smoker, number (\%) & $90(42)$ & $20(47)$ & $70(40)$ & \\
\hline Former smoker, number (\%) & $109(51)$ & $20(47)$ & $89(51)$ & \\
\hline Current smoker, number (\%) & $17(8)$ & $2(5)$ & $15(9)$ & \\
\hline Body Mass Index (Mean \pm SD) & $26.0 \pm 4.4$ & $26.1 \pm 5.1$ & $26.0 \pm 4.2$ & $0.967^{c}$ \\
\hline Taking Cardiovascular Drugs, number (\%) & $156(72)$ & $28(65)$ & $128(74)$ & $0.370^{b}$ \\
\hline Taking Vitamins, number (\%) & $49(23)$ & $3(7)$ & $46(26)$ & $0.007^{b}$ \\
\hline Takin low-dose aspirin, number (\%) & $73(34)$ & $5(12)$ & $68(39)$ & $0.001^{b}$ \\
\hline
\end{tabular}

${ }^{a}$ Mann Whitney $U$ test

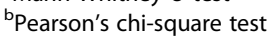

'Independent sample t-test

Therefore, this was not included in the subsequent analysis of confounders.

\section{Plasma LCN2, MMP9 and MMP9/LCN2 in nAMD patients}

The plasma level of LCN2 in nAMD patients was $181.46 \pm 73.62 \mathrm{ng} / \mathrm{ml}$, significantly higher than that in healthy controls $(152.24 \pm 49.55 \mathrm{ng} / \mathrm{ml}, P=0.047$, independent samples test, Table 2). However, the difference became insignificant after correcting for age or taking low-dose aspirin $(p=0.259$ and 0.602 , respectively, Table $2)$. A linear regression study uncovered a positive correlation between age and the plasma levels of LCN2 in nAMD patients $(r=0.29, p=0.0002$, Fig. 1a, red line and red dots). No statistically significant correlation $(r=0.28$, $p=0.06$. Figure $1 \mathrm{a}$, green line and green dots) was observed in healthy controls. Our results suggest that plasma levels of LCN2 were positively affected by age, particularly in nAMD patients. No correlation was observed between the plasma levels of LCN2 and the number of intravitreal Lucentis injection in nAMD patients (Fig. 1b).

There was no difference in the plasma levels of MMP9 and LCN2/MMP9 complex between nAMD patients and healthy controls (Table 2).

We previously observed a higher population of circulating neutrophils in nAMD patients [19]. The result was confirmed in this cohort of participants (Fig. 2a). To understand if higher numbers of circulating neutrophils contribute to increased LCN2, a correlation analysis was conducted in the participants whose neutrophil data were collected in our study [19]. As expected, the population of circulating neutrophils was positively correlated with plasma levels of LCN2 in nAMD patients $(r=0.34, p=0.0007$, Fig. $2 \mathrm{~b})$ but not in healthy controls $(r=0.057, p=0.77$,

Table 2 Plasma levels of LCN2, MMP9 and MMP9/LCN2 complex in nAMD and controls

\begin{tabular}{lllllll}
\hline $\begin{array}{l}\text { Variable } \\
(\mathbf{n g} / \mathbf{m l})\end{array}$ & $\begin{array}{l}\text { Control } \\
(\text { Mean } \pm \text { SD })\end{array}$ & $\begin{array}{l}\text { nAMD } \\
(\text { Mean } \pm \text { SD })\end{array}$ & $\begin{array}{l}\text { Univariate analysis } \\
(\boldsymbol{p} \text { value })\end{array}$ & $\begin{array}{l}\text { Corrected by age } \\
(\boldsymbol{p} \text { value })\end{array}$ & $\begin{array}{l}\text { Corrected by taking } \\
\text { aspirin }(\boldsymbol{p} \text { value })\end{array}$ & $\begin{array}{l}\text { Corrected by taking } \\
\text { aspirin and age }\end{array}$ \\
\hline LCN2 & $N=43$ & $N=168$ & & $0.259^{\mathrm{b}}$ & $0.602^{\mathrm{c}}$ & $0.396^{\mathrm{d}}$ \\
& $153.24 \pm 49.55$ & $181.46 \pm 73.62$ & $0.047^{\mathrm{a}}$ & & \\
MMP9 & $N=43$ & $N=167$ & & & \\
& $124.16 \pm 63.12$ & $116.36 \pm 68.45$ & $0.274^{\mathrm{a}}$ & & \\
MMP9/ & $N=43$ & $N=167$ & & & \\
LCN2 & $188.38 \pm 45.12$ & $179.16 \pm 58.11$ & $0.335^{\mathrm{a}}$ & &
\end{tabular}

Independent samples t test

bunivariate analysis corrected for age

'Univariate analysis corrected for taking aspirin

dUnivariate analysis corrected for age and taking aspirin 


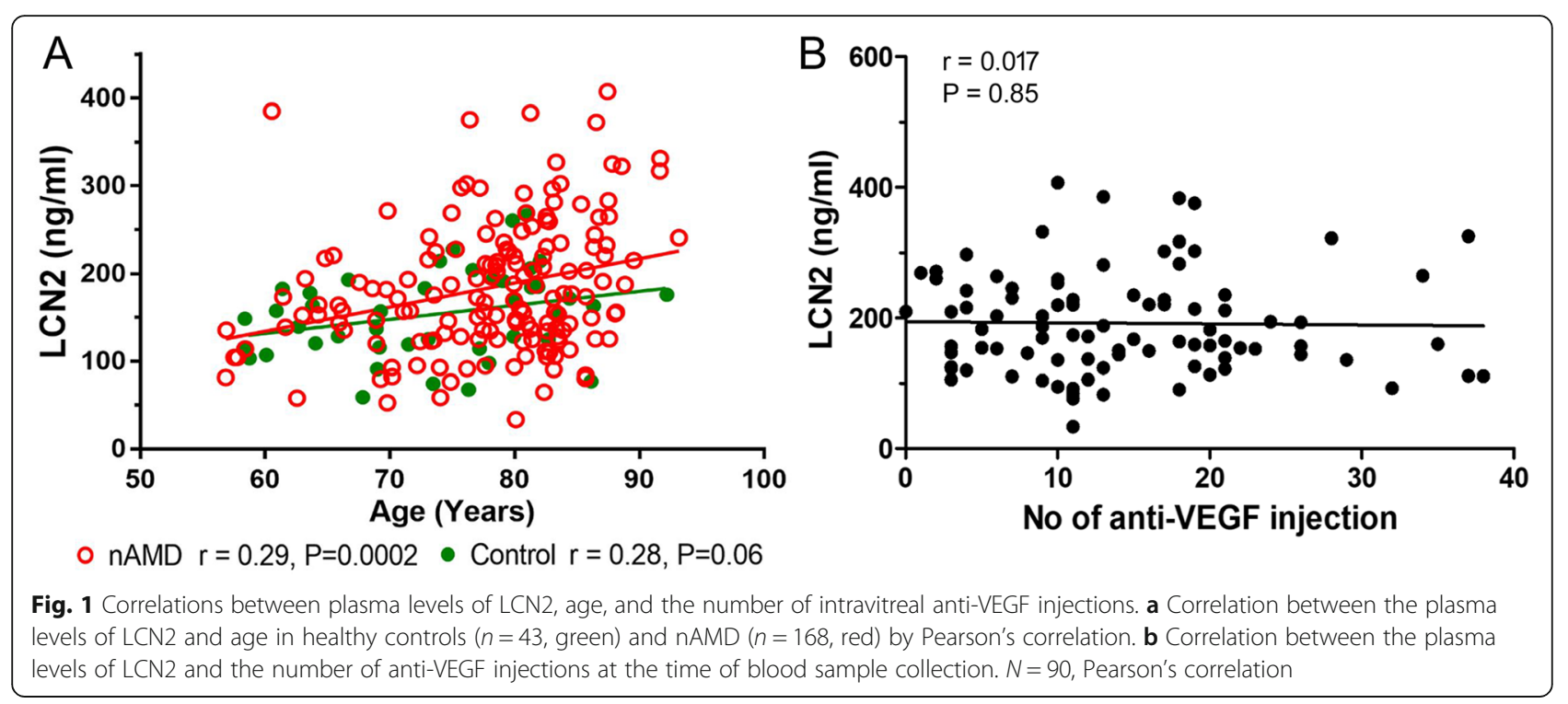

Fig. 2c). Interestingly, no correlation was observed between age and circulating neutrophils in both nAMD patients (Fig. 2d) and controls (Fig. 2e), suggesting that ageing and circulating neutrophils contribute independently to the increased plasma levels of LCN2 in nAMD patients.
LCN2 in nAMD subgroups

Out of the 174 nAMD participants, 108 had CNV, 32 with RAP, 23 with PCV and 11 were unknown. One-way ANOVA analysis did not reveal any significant difference in the plasma levels of LCN2, MMP9 or MMP9/LCN2 complex among the control, CNV, RAP and PVC groups

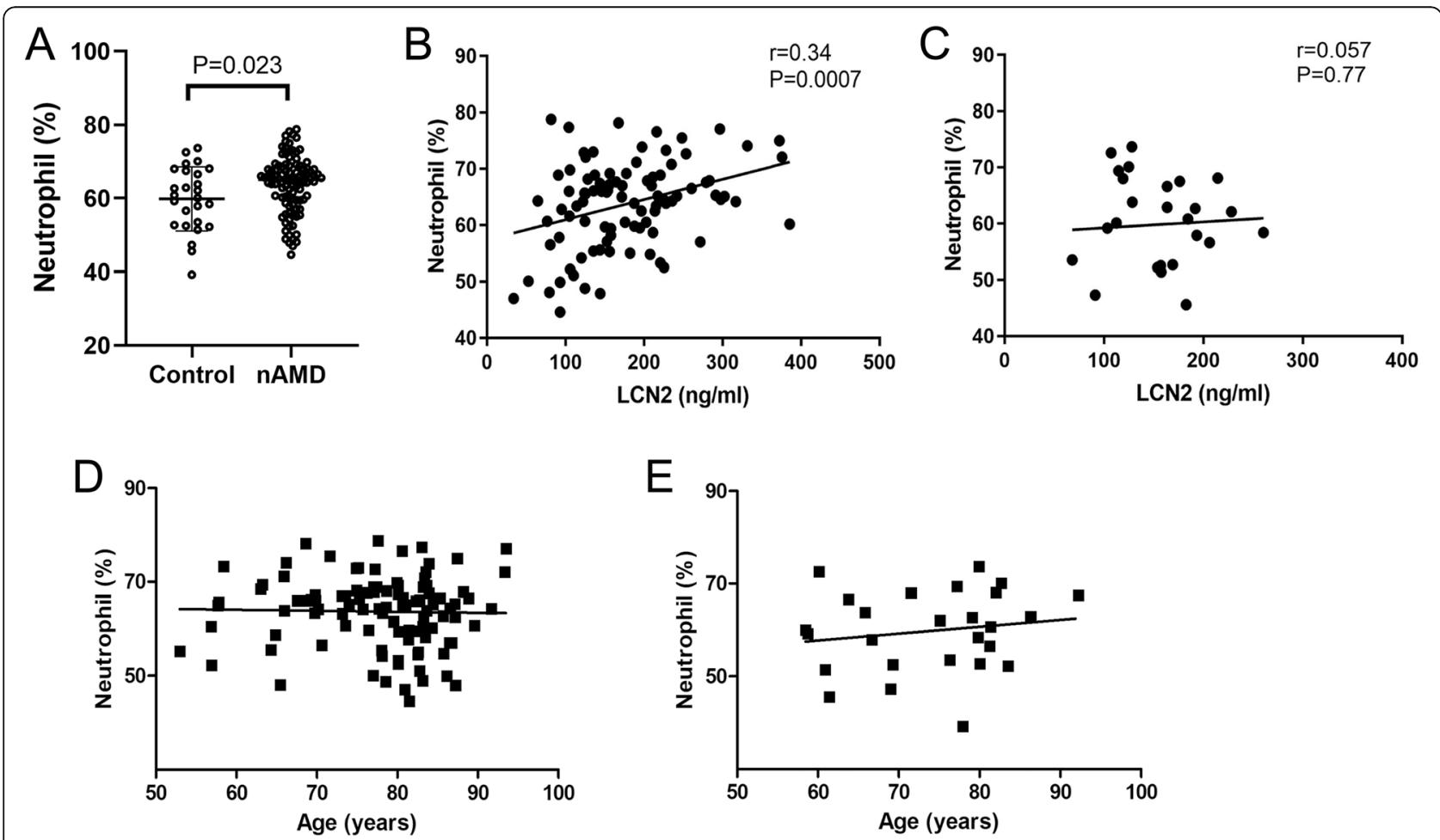

Fig. 2 Correlations between circulating neutrophils, plasma levels of LCN2 and age. Neutrophils were identified by flow cytometry of peripheral blood as CD11 b ${ }^{+} \mathrm{CD} 16^{+} \mathrm{HLA}-\mathrm{DR} \mathrm{R}^{-}$, and plasma levels of LCN2 were measured by ELISA. a Neutrophils in healthy controls and nAMD. Unpaired ttest. b, c Correlations between the plasma levels of LCN2 and circulating neutrophils in nAMD (b) and healthy controls (c). d, e Correlations between circulating neutrophils and age in $\operatorname{nAMD}(\mathbf{d})$ and healthy controls (e). nAMD: $n=71$, Healthy control: $n=26$. Pearson's correlation 
( $p=0.108,0.301$ and 0.370 respectively, Table 3$)$. Univariate analysis showed that the plasma levels of LCN2 in CNV subgroup were higher than those of control group ( $p=0.006$, Table 3 ), however, the difference became insignificant after adjusting for age $(p=0.064)$.

LCN2 in nAMD patients with or without macular fibrosis Out of the 174 nAMD participants, 58 had macular fibrosis and 110 did not have fibrosis and 6 were unknown. One-way ANOVA showed a significant difference in the plasma levels of LCN2 among the three groups (controls, nAMD with fibrosis, nAMD without fibrosis, $p=0.048$ ), with the highest levels detected in patients with macular fibrosis $(192.77 \pm 76.37 \mathrm{ng} / \mathrm{ml}$, Fig. 3). Post hoc Tukey test suggested that the difference resided between the groups of controls and nAMD with fibrosis $(p=0.037$, Fig. 3$)$. The mean age of the control group $(73.4 \pm 8.9)$ was significantly younger than that of fibrosis group $(79 \pm 9.2)$ and non-fibrosis group (77.9 \pm 8.1) ( $p=0.002$, and 0.013 respectively). After adjusting for age, the difference between nAMD with macular fibrosis and controls remains significant ( $p=0.033$, Fig. 3$)$.

\section{LCN2 and responsiveness to anti-VEGF therapy or Geographic Atrophy (GA)}

Out of the 174 nAMD patients, 80 (45.98\%) patients responded completely, 90 (51.72\%) responded partially, and $4(2.30 \%)$ did not respond to the anti-VEGF therapy. Due to the limited number of non-responders in this study, this group was not included in the statistical analysis. There was no significant difference in the plasma levels of LCN2 between controls $(154.21 \pm 49.74 \mathrm{ng} / \mathrm{ml})$ and partial responders $(178.82 \pm 72.35 \mathrm{ng} / \mathrm{ml})$ or complete responders $(183.90 \pm 75.44 \mathrm{ng} / \mathrm{ml})$. In addition, the plasma levels of LCN2 between patients with GA $(181.52 \pm 73.03 \mathrm{ng} / \mathrm{ml}, n=80)$ and those without GA $(178.74 \pm 68.69 \mathrm{ng} / \mathrm{ml}, n=34)$ were comparable.

\section{Discussion}

LCN2 is known to be produced predominately by neutrophils in the immune system [27]. Previously, we and others have shown that nAMD patients had a higher

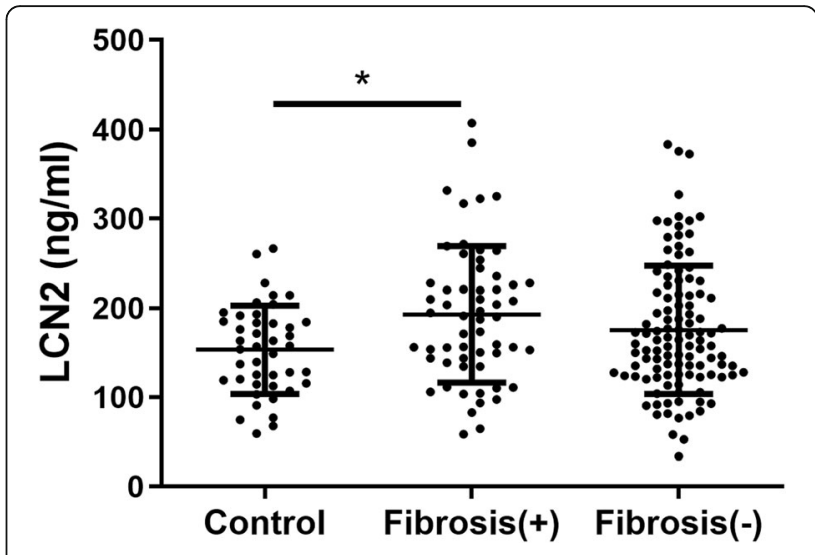

Fig. 3 Plasma levels of LCN2 in healthy control, nAMD patients with or without macular fibrosis. Plasma LCN2 were measured by ELISA. Healthy control: $n=43$; Fibrosis (+): $n=58$; Fibrosis $(-): n=110$. ${ }^{*} P=$ 0.033, univariate analysis corrected for age

percentage of circulating neutrophils compared to healthy controls $[18,19,25,41]$. In this study, we observed a positive correlation between plasma levels of LCN2 and circulating neutrophils in nAMD patients but not in healthy controls (Fig. 2c-d). Our results suggest that higher plasma levels of LCN2 in nAMD patients may, at least in part, attribute to circulating neutrophils. Further studies will be needed to understand the functional difference between neutrophils from nAMD and healthy controls and whether LCN2 is produced or upregulated by a specific subset of neutrophils in nAMD. Interestingly, we also observed a positive correlation between plasma levels of LCN2 and age in nAMD patients but not in controls (Fig. 1a), and there was no correlation between neutrophil and age (Fig. 2d-e), indicating that ageing may affect plasma levels of LCN2 in nAMD independent of circulating neutrophils. Together, our results suggest nAMD patients have abnormal ageing in terms of LCN2 production.

In addition to neutrophils, LCN2 can also be produced by various tissue cells particularly under disease conditions. Plasma and urine levels of LCN2 have been used as a biomarker for kidney disease [42, 43] and cancer progression [44]. Recent studies have shown that retinal

Table 3 Plasma levels of LCN2, MMP9, and MMP9/LCN2 complex in different types of nAMD patients and controls

\begin{tabular}{|c|c|c|c|c|c|}
\hline Variable & Control $(N=43$, Mean $\pm S D)$ & CNV $(N=108$, Mean $\pm S D)$ & $\operatorname{RAP}(N=32$, Mean \pm SD $)$ & $\operatorname{PCV}(N=23$, Mean \pm SD $)$ & $p$ value \\
\hline Age & $73.4 \pm 8.9$ & $78.9 \pm 7.8^{\dagger}$ & $80.7 \pm 8.7^{\dagger}$ & $71.6 \pm 8.9$ & \\
\hline LCN2 (ng/ml) & $153.24 \pm 49.55$ & $186.60 \pm 71.57^{*}$ & $176.08 \pm 82.70$ & $168.36 \pm 70.68$ & $0.108^{\mathrm{a}}$ \\
\hline MMP9 (ng/ml) & $124.16 \pm 63.12$ & $114.42 \pm 58.93$ & $111.18 \pm 96.94$ & $126.40 \pm 65.09$ & $0.301^{a}$ \\
\hline MMP9/LCN2 (ng/ml) & $188.38 \pm 45.12$ & $176.51 \pm 53.35$ & $181.69 \pm 66.07$ & $196.29 \pm 66.05$ & $0.370^{b}$ \\
\hline
\end{tabular}

CNV choroidal neovascularisation, RAP retinal angiomatous proliferation, $P C V$ polypoidal choroidal vasculopathy

${ }^{\dagger} P<0.05$ compared to controls

${ }^{*} P=0.006$ compared to controls in univariate analysis

${ }^{a}$ Kruskal-Wallis one-way analysis of variance on log transformed data

bone-way ANOVA 
cells including Müller glia [45-47], microglia and RPE $[47,48]$ can all express LCN2 and the expression is upregulated under stress conditions [45, 47, 49]. Furthermore, increased plasma levels of LCN2 have been observed in patients with Stargardt disease, retinitis pigmentosa, and AMD [46]. The diseased macula in nAMD may release LCN2 to the blood circulation contributing to higher plasma levels of this protein.

In this study, the plasma levels of free LCN2 and MMP9/LCN2 complex were comparable in nAMD patients and MMP9 was not significantly increased in nAMD, suggesting that the free form of LCN2, but not the MMP9/LCN2 complex or MMP9 is involved in nAMD. The role of LCN2 in retinal diseases remains controversial. It has been reported that LCN2 participates in light-induced photoreceptor death [50] and the development of early AMD [51]. However, other studies have shown that LCN2 is also involved in retinal pro-survival and anti-oxidative responses [46] and can suppress inflammation and reduce retinal degeneration $[45,46]$. In this study, we observed a significantly higher plasma level of LCN2 in nAMD patients with macular fibrosis, suggesting its potential pro-fibrotic roles. LCN2 is reported to contribute to cardiovascular [32] and kidney [31, 52] fibrosis and promote epithelial-to-mesenchymal transition (EMT) in breast cancers [39]. LCN2 is also known to have antifibrotic roles as overexpression of LCN2 reduced kidney [34] and liver [33] fibrosis and suppressed epidermal growth factor (EGF) or transforming growth factor-beta (TGF $\beta$ )-induced EMT [53]. In addition, LCN2 can bind and stabilise MMP9, which is critically involved in extracellular matrix remodelling during inflammation and wound healing. The role of LCN2 in macular fibrosis warrants further investigation.

The strengths of this study include independent grading of nAMD subtypes, fibrosis, GA and anti-VEGF responsiveness, and extensive exploration of changes in the plasma levels of LCN2, MMP9 and LCN2/MMP9 complex in different types of nAMD as well as in patients partially or completely responding to anti-VEGF treatment. Limitations of the current study include relatively small sample size particularly in subgroups of patients (i.e. RAP $n=32$, PCV $n=23$ ), the recruitment of all participants from one location, and uncontrolled age in control and nAMD groups. In addition, participants were recruited to the study at different times after diagnosis of nAMD, and some patients enrolled into the study at the early stages of nAMD and classified as having no fibrosis or no GA might still develop fibrosis or GA at later time points.

\section{Conclusions}

We demonstrated that the plasma level of LCN2 was positively correlated with age and the percentage of circulating neutrophils in nAMD patients. Higher plasma levels of LCN2 may contribute to the development of macular fibrosis.

\section{Methods \\ Study participants}

The study protocol was approved by the Research Ethics Committee of Queen's University Belfast and procedures were followed the tenets of the Declaration of Helsinki on research into human volunteers. Recruitment criteria for participants include: 1) older than 50 years old, 2) don't have systemic inflammatory or autoimmune disorders (for examples with active chronic bronchitis, rheumatoid arthritis); 3) are not undergoing steroid therapy or chemotherapy. The nAMD participants were recruited from patients who attended the Macular disease clinics in Belfast Health and Social Care Trust, UK. The healthy controls were accompanying persons of the nAMD patients, who were either spouses, relatives or friends. Written informed consent was obtained from every participant. Participant's information was ascertained using a structured questionnaire including medical history, current medication, family history of AMD, smoking habits (current, ex-smoker and never-smoker) and body mass index (BMI).

The eye conditions of healthy controls were confirmed by clinical examination via fundus photography and spectral domain-optical coherence tomography (SD-OCT). nAMD were diagnosed by clinical examination of colour fundus photography (CFP), autofluorescence, SD-OCT, fluorescein angiography and indocyanine green angiography. nAMD subgroups including $\mathrm{CNV}$, RAP and PCV were identified at the time of recruitment. Participants with nAMD received intravitreal anti-VEGF treatment (Lucentis) and were followed up for over 6 months. Their fundus colour images and tomographic scans were graded at baseline and their most recent hospital visit prior to closure of the database. Responsiveness to treatment was defined based on the participant achieving a fluid-free macula at any stage during follow-up in the treated eye. In addition, the status of whether a patient was fluid free at the month3 and month-6 examinations was also recorded. Participants were classified into the following three categories: (1) complete responder: resolution of leakage at any time point during follow up; (2) partial responder: exhibiting dependence on VEGF inhibitors but a fluid-free macula never achieved; and (3) non-responder: no morphological improvement or worsening. Macular scar identification was based on both CFP and OCT characteristics. On CFP, macular scar was defined as well-delineated areas of yellowish-white tissue, which on OCT corresponded to the presence of linear bands of hyper reflective material that had either obscured or replaced the normal hyper reflective bands of the neurosensory retina and RPE/Bruch's membrane complex. Macular atrophy (MA) was defined as 
single or multiple areas of hypopigmentation with welldefined borders and visible large choroidal vessels on CFP, which corresponded to window defects on angiography and/ or to the loss of cellular layers (outer retina, RPE and choriocapillaris) on the accompanying tomograms [12, 19, 20].

\section{Sample collection}

Peripheral blood samples were drawn into EDTA treated tubes and transferred to the research lab at room temperature. Plasma was extracted within $3 \mathrm{~h}$ after blood collection. Plasma was first separated from the whole blood with centrifugation of $10 \mathrm{~min}$ at $300 \mathrm{~g}$ and followed by further centrifugation of $10 \mathrm{~min}$ at $2000 \mathrm{~g}$ to remove cell debris or platelets. Plasma aliquots were stored at $-80^{\circ} \mathrm{C}$ until analysis.

\section{Flow cytometry analysis of neutrophils}

Circulating neutrophils were identified by flow cytometry using the protocol described in our previous publications $[19,54]$. In brief, $30 \mu \mathrm{l}$ of fresh blood were incubated with fluorochrome-labelled antibody cocktail (CD19-FITC, CD16-Pacific Blue, CD11b-APC (all from BD Biosciences, Oxford, UK) and HLA-DR-PE (eBioscience, UK)) for 45 min in the dark at $4{ }^{\circ} \mathrm{C}$. Red blood cells were removed with lysis buffer (BD Biosciences) and samples were acquired using a BD Canto II flow cytometer (BD Biosciences). The flow cytometry data were analysed using FlowJo (version 10, Tree Star Inc., Ashland, OR, USA). Neutrophils were identified based on their cell size (FSC) and granularity (SSC) as well as their cell surface antigens

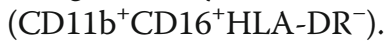

\section{Enzyme-Linked Immunosorbent Assay (ELISA)}

LCN2, MMP9 and MMP9/LCN2 complex DuoSet ELISA kits were purchased from $\mathrm{R} \& \mathrm{D}$ systems (Abingdon, Oxford, UK). The ELISAs were performed following the protocols provided by the manufacture. The results were measured using a plate reader at the wavelength of $450 \mathrm{~nm}$ and correction at $560 \mathrm{~nm}$. A standard curve was generated using GraphPad following a fourparameter logistic (4-PL) curve-fit.

\section{Statistical analysis}

Statistical analysis was performed using the Statistical Package for the Social Sciences (SPSS), window version 25 (the International Business Machines Corporation, IBM). All continuous variables were firstly tested for normality using the Kolmogorov-Smirnov test. Independent-Samples t-test or One-way ANOVA were used to analyse normally distributed continuous variables; whereas nonparametric test including MannWhitney U test (for two samples) and Kruskal-Wallis one-way ANOVA (for more than two samples) were used to examine continuous variables which were not normally distributed. Pearson's Chi-square test was used to examine categorical variables such as demographic information and clinical data. Bivariate correlation was performed using Pearson's correlation. Univariate analysis was performed to examine associations of variables. Data were presented as mean $\pm \mathrm{SD}, p$ value $<0.05$ was considered as statistically significant in all cases.

\section{Abbreviations \\ AMD: Age-related macular degeneration; BMl: Body mass index; \\ CNV: Choroidal neovascularisation; EMT: Epithelial-to-mesenchymal transition; nAMD: Neovascular age-related macular degeneration; GA: Geographic atrophy; LCN2: Lipocalin-2; MMP9: Matrix metalloproteinase 9; PCV: Polypoidal choroidal vasculopathy; RAP: Retinal angiomatous proliferation; RPE: Retinal pigment epithelium; SD-OCT: Spectral domain optical coherence tomography; VEGF: Vascular endothelial growth factor}

\section{Acknowledgements}

We thank Research nurses Rebecca Denham and Georgina Sterrett for their help in patient recruitment. We thank the patients who participated in this study. We thank Dr. David Wright for helpful discussions during data analysis.

\section{Authors' contributions}

$\mathrm{MC}$ and HX conceived and designed the experiments, wrote the paper. MC, $N Y$ and $J$ conducted the experiments. MC, NY, REH, HX analysed the results. LT, GS and UC conducted clinical analysis and recruited patients. All authors read and approved the final manuscript.

\section{Funding}

The study was funded by the Dunhill Medical Trust (R188/0211) and Guide Dogs for the Blindness Association UK (2008-5a). The funding bodies have no role in the design of the study, collection, analysis and interpretation of data, and in writing the manuscript.

\section{Availability of data and materials}

The datasets generated and analysed during the current study are available from the corresponding author on reasonable request.

Ethics approval and consent to participate

The study was approved by the Research Ethics Committee of Queen's University Belfast and consent was obtained from each participant.

Consent for publication

Not applicable.

Competing interests

The authors declare that they have no competing interests.

\section{Author details}

${ }^{1}$ Centre for Experimental Medicine, The Wellcome-Wolfson Institute for Experimental Medicine, School of Medicine, Dentistry \& Biomedical Science, Queen's University Belfast, 97 Lisburn Road, Belfast BT9 7BL, UK. ${ }^{2}$ Centre for Public Health, School of Medicine, Dentistry \& Biomedical Science, Queen's University Belfast, 97 Lisburn Road, Belfast BT9 7BL, UK. ${ }^{3}$ Belfast Health and Social Care Trust, Belfast BT12 6BA, UK.

Received: 15 July 2020 Accepted: 1 November 2020

Published online: 14 November 2020

\footnotetext{
References

1. Wong WL, Su X, Li X, Cheung CM, Klein R, Cheng CY, et al. Global prevalence of age-related macular degeneration and disease burden projection for 2020 and 2040: a systematic review and meta-analysis. Lancet Glob Health. 2014;2(2):e106-16.

2. Chakravarthy U, Evans J, Rosenfeld PJ. Age related macular degeneration. Bmj. 2010;340:c981.
} 
3. Yannuzzi LA, Negrao S, lida T, Carvalho C, Rodriguez-Coleman H, Slakter J, et al. Retinal angiomatous proliferation in age-related macular degeneration. Retina (Philadelphia, Pa). 2001;21(5):416-34.

4. Ciardella AP, Donsoff IM, Huang SJ, Costa DL, Yannuzzi LA. Polypoidal choroidal vasculopathy. Surv Ophthalmol. 2004;49(1):25-37.

5. Lim LS, Mitchell P, Seddon JM, Holz FG, Wong TY. Age-related macular degeneration. Lancet. 2012;379(9827):1728-38.

6. Holz FG, Schmitz-Valckenberg S, Fleckenstein M. Recent developments in the treatment of age-related macular degeneration. J Clin Invest. 2014 124(4):1430-8.

7. Rofagha S, Bhisitkul RB, Boyer DS, Sadda SR, Zhang K, Group S-US. Seven-year outcomes in ranibizumab-treated patients in ANCHOR, MARINA, and HORIZON: a multicenter cohort study (SEVEN-UP). Ophthalmology. 2013;120(11):2292-9.

8. Daniel E, Toth CA, Grunwald JE, Jaffe GJ, Martin DF, Fine SL, et al. Risk of scar in the comparison of age-related macular degeneration treatments trials. Ophthalmology. 2014;121(3):656-66.

9. Chen M, Xu H. Parainflammation, chronic inflammation, and age-related macular degeneration. J Leukoc Biol. 2015;98:713-25.

10. Ambati J, Atkinson JP, Gelfand BD. Immunology of age-related macular degeneration. Nat Rev Immunol. 2013;13(6):438-51.

11. Schick T, Steinhauer M, Aslanidis A, Altay L, Karlstetter M, Langmann T, et al. Local complement activation in aqueous humor in patients with agerelated macular degeneration. Eye (London, England). 2017:31(5):810-3.

12. Lechner J, Chen M, Hogg RE, Toth L, Silvestri G, Chakravarthy U, et al. Higher plasma levels of complement C3a, C4a and C5a increase the risk of subretinal fibrosis in neovascular age-related macular degeneration: Complement activation in AMD. Immun Ageing. 2016;13 4-016-0060-5. eCollection 2016.

13. McHarg S, Clark SJ, Day AJ, Bishop PN. Age-related macular degeneration and the role of the complement system. Mol Immunol. 2015;67(1):43-50.

14. Whitmore SS, Sohn EH, Chirco KR, Drack AV, Stone EM, Tucker BA, et al. Complement activation and choriocapillaris loss in early AMD: implications for pathophysiology and therapy. Prog Retin Eye Res. 2015;45:1-29.

15. Warwick A, Khandhadia S, Ennis S, Lotery A. Age-related macular degeneration: a disease of systemic or local complement Dysregulation? J Clin Med. 2014;3(4):1234-57.

16. Subhi $Y$, Nielsen MK, Molbech CR, Liisborg C, Søndergaard HB, Sellebjerg $F$, et al. The transcriptome of peripheral blood mononuclear cells in patients with clinical subtypes of late age-related macular degeneration. Immun Ageing. 2019:16:20.

17. Subhi Y, Krogh Nielsen M, Molbech CR, Krüger Falk M, Singh A, Hviid TVF, et al. Association of CD11b+ monocytes and anti-vascular endothelial growth factor injections in treatment of Neovascular age-related macular degeneration and Polypoidal Choroidal vasculopathy. JAMA Ophthalmol. 2019;137(5):515-22.

18. Krogh Nielsen M, Hector SM, Allen K, Subhi Y, Sørensen TL. Altered activation state of circulating neutrophils in patients with neovascular agerelated macular degeneration. Immun Ageing. 2017;14:18,

19. Lechner J, Chen M, Hogg RE, Toth L, Silvestri G, Chakravarthy U, et al. Alterations in circulating immune cells in Neovascular age-related macular degeneration. Sci Rep. 2015;5:16754

20. Lechner J, Chen M, Hogg RE, Toth L, Silvestri G, Chakravarthy U, et al. Peripheral blood mononuclear cells from neovascular age-related macular degeneration patients produce higher levels of chemokines CCL2 (MCP-1) and CXCL8 (IL-8). J Neuroinflammation. 2017;14(1):42 017-0820-y

21. Nassar K, Grisanti S, Elfar E, Luke J, Luke M, Grisanti S. Serum cytokines as biomarkers for age-related macular degeneration. Graefes Arch Clin Exp Ophthalmol. 2015;253(5):699-704.

22. Grunin M, Burstyn-Cohen T, Hagbi-Levi S, Peled A, Chowers I. Chemokine receptor expression in peripheral blood monocytes from patients with neovascular age-related macular degeneration. Invest Ophthalmol Vis Sci. 2012;53(9):5292-300

23. Miao H, Tao Y, Li XX. Inflammatory cytokines in aqueous humor of patients with choroidal neovascularization. Mol Vis. 2012;18:574-80.

24. Nahavandipour A, Krogh Nielsen M, Sørensen TL, Subhi Y. Systemic levels of interleukin-6 in patients with age-related macular degeneration: a systematic review and meta-analysis. Acta Ophthalmol. 2020.

25. Ithan N, Daglioglu MC, Ithan O, Coskun M, Tuzcu EA, Kahraman H, et al. Assessment of neutrophil/lymphocyte ratio in patients with age-related macular degeneration. Ocul Immunol Inflamm. 2014:1-4.
26. Ozgonul C, Sertoglu E. Accurate use of neutrophil/lymphocyte ratio in patients with age-related macular degeneration. Ocul Immunol Inflamm. 2014:1-2.

27. Flower DR. The lipocalin protein family: structure and function. Biochem J. 1996:318(Pt 1):1-14

28. Rodvold JJ, Mahadevan NR, Zanetti M. Lipocalin 2 in cancer: when good immunity goes bad. Cancer Lett. 2012:316(2):132-8.

29. Yan L, Borregaard N, Kjeldsen L, Moses MA. The high molecular weight urinary matrix metalloproteinase (MMP) activity is a complex of gelatinase B/MMP-9 and neutrophil gelatinase-associated lipocalin (NGAL). Modulation of MMP-9 activity by NGAL. J Biol Chem. 2001;276(40):37258-65.

30. Chau KY, Sivaprasad S, Patel N, Donaldson TA, Luthert PJ, Chong NV. Plasma levels of matrix metalloproteinase-2 and -9 (MMP-2 and MMP-9) in agerelated macular degeneration. Eye (Lond). 2008;22(6):855-9.

31. Entin-Meer M, Ben-Shoshan J, Maysel-Auslender S, Levy R, Goryainov P, Schwartz I, et al. Accelerated renal fibrosis in cardiorenal syndrome is associated with long-term increase in urine neutrophil gelatinase-associated lipocalin levels. Am J Nephrol. 2012;36(2):190-200.

32. Tarjus A, Martínez-Martínez E, Amador C, Latouche C, El Moghrabi S, Berger $T$, et al. Neutrophil Gelatinase-associated Lipocalin, a novel mineralocorticoid biotarget, mediates vascular Profibrotic effects of mineralocorticoids. Hypertension. 2015:66(1):158-66.

33. Aoyama T, Kuwahara-Arai K, Uchiyama A, Kon K, Okubo H, Yamashina S, et al. Spleen-derived lipocalin-2 in the portal vein regulates Kupffer cells activation and attenuates the development of liver fibrosis in mice. Lab Investig. 2017;97(8):890-902.

34. Guiteras R, Sola A, Flaquer M, Hotter G, Torras J, Grinyó JM, et al. Macrophage overexpressing NGAL ameliorated kidney fibrosis in the UUO mice model. Cell Physiol Biochem. 2017;42(5):1945-60.

35. Stanski N, Menon S, Goldstein SL, Basu RK. Integration of urinary neutrophil gelatinase-associated lipocalin with serum creatinine delineates acute kidney injury phenotypes in critically ill children. J Crit Care. 2019;53:1-7.

36. Viau A, El Karoui K, Laouari D, Burtin M, Nguyen C, Mori K, et al. Lipocalin 2 is essential for chronic kidney disease progression in mice and humans. J Clin Invest. 2010;120(11):4065-76.

37. Al Nimer F, Elliott C, Bergman J, Khademi M, Dring AM, Aeinehband S, et al. Lipocalin-2 is increased in progressive multiple sclerosis and inhibits remyelination. Neurol Neuroimmunol Neuroinflamm. 2016;3(1):e191.

38. Bi F, Huang C, Tong J, Qiu G, Huang B, Wu Q, et al. Reactive astrocytes secrete Icn2 to promote neuron death. Proc Natl Acad Sci U S A. 2013; 110(10):4069-74.

39. Yang J, Bielenberg DR, Rodig SJ, Doiron R, Clifton MC, Kung AL, et al. Lipocalin 2 promotes breast cancer progression. Proc Natl Acad Sci U S A. 2009;106(10):3913-8.

40. Ghosh S, Stepicheva N, Yazdankhah M, Shang P, Watson AM, Hose S, et al. The role of lipocalin-2 in age-related macular degeneration (AMD). Cell Mol Life Sci. 2020;77(5):835-51.

41. Niazi S, Krogh Nielsen M, Sørensen TL, Subhi Y. Neutrophil-to-lymphocyte ratio in age-related macular degeneration: a systematic review and metaanalysis. Acta Ophthalmol. 2019:97(6):558-66.

42. Goldstein SL. Urinary NGAL to define AKI in asphyxiated infants. Pediatr Nephrol. 2015;30(7):1047-9.

43. Forster CS, Loechtenfeldt AM, Shah SS, Goldstein S. Urine neutrophil gelatinase-associated lipocalin in girls with recurrent urinary tract infections. Pediatr Nephrol. 2020;35:2121-8.

44. Yang J, Moses MA. Lipocalin 2: a multifaceted modulator of human cancer. Cell Cycle. 2009;8(15):2347-52.

45. Tang W, Ma J, Gu R, Ding X, Lei B, Wang X, et al. Lipocalin 2 suppresses ocular inflammation by inhibiting the activation of NF-K $\beta$ pathway in endotoxin-induced uveitis. Cell Physiol Biochem. 2018; 46(1):375-88.

46. Parmar T, Parmar VM, Perusek L, Georges A, Takahashi M, Crabb JW, et al. Lipocalin 2 plays an important role in regulating inflammation in retinal degeneration. J Immunol. 2018;200(9):3128-41.

47. Parmar T, Parmar VM, Arai E, Sahu B, Perusek L, Maeda A. Acute stress responses are early molecular events of retinal degeneration in $\mathrm{Abca} 4$ -/-Rdh8-/- mice after light exposure. Invest Ophthalmol Vis Sci. 2016; 57(7):3257-67.

48. Valapala M, Edwards M, Hose S, Grebe R, Bhutto IA, Cano M, et al. Increased Lipocalin-2 in the retinal pigment epithelium of Cryba1 CKO mice is associated with a chronic inflammatory response. Aging Cell. 2014;13(6): $1091-4$. 
49. Ghosh S, Shang P, Yazdankhah M, Bhutto I, Hose S, Montezuma SR, et al. Activating the AKT2-nuclear factor-KB-lipocalin-2 axis elicits an inflammatory response in age-related macular degeneration. J Pathol. 2017;241(5):583-8.

50. Tang W, Ma J, Gu R, Lei B, Ding X, Xu G. Light-induced Lipocalin 2 facilitates cellular apoptosis by positively regulating reactive oxygen species/Bim signaling in retinal degeneration. Invest Ophthalmol Vis Sci. 2018:59(15): 6014-25.

51. Ghosh S, Padmanabhan A, Vaidya T, Watson AM, Bhutto IA, Hose S, et al. Neutrophils homing into the retina trigger pathology in early age-related macular degeneration. Commun Biol. 2019;2:348.

52. Kashiwagi E, Tonomura Y, Kondo C, Masuno K, Fujisawa K, Tsuchiya N, et al. Involvement of neutrophil gelatinase-associated lipocalin and osteopontin in renal tubular regeneration and interstitial fibrosis after cisplatin-induced renal failure. Exp Toxicol Pathol. 2014;66(7):301-11.

53. Wang YP, Yu GR, Lee MJ, Lee SY, Chu IS, Leem SH, et al. Lipocalin-2 negatively modulates the epithelial-to-mesenchymal transition in hepatocellular carcinoma through the epidermal growth factor (TGF-beta1)/ Lcn2/Twist1 pathway. Hepatology. 2013;58(4):1349-61.

54. Obasanmi G, Lois N, Armstrong D, Lavery NJ, Hombrebueno JR, Lynch A, et al. Circulating leukocyte alterations and the development/progression of diabetic retinopathy in type 1 diabetic patients - a pilot study. Curr Eye Res. 2020:1-11.

\section{Publisher's Note}

Springer Nature remains neutral with regard to jurisdictional claims in published maps and institutional affiliations.

Ready to submit your research? Choose BMC and benefit from:

- fast, convenient online submission

- thorough peer review by experienced researchers in your field

- rapid publication on acceptance

- support for research data, including large and complex data types

- gold Open Access which fosters wider collaboration and increased citations

- maximum visibility for your research: over $100 \mathrm{M}$ website views per year

At $\mathrm{BMC}$, research is always in progress.

Learn more biomedcentral.com/submissions 